\title{
DTMOS Mode as an Effective Solution of RTN Suppression for Robust Device/Circuit Co-Design
}

\author{
Shaofeng Guo ${ }^{1}$, Ru Huang ${ }^{1,3,}$, Peng Hao ${ }^{1}$, Mulong Luo ${ }^{1}$, Pengpeng Ren ${ }^{1}$, Jianping Wang ${ }^{2}$, Weihai $\mathrm{Bu}^{2}$, Jingang $\mathrm{Wu}^{2}$, \\ Waisum Wong ${ }^{2,3}$, Scott $\mathrm{Yu}^{2,3}$, Hanming $\mathrm{Wu}^{2,3}$, Shiuh-Wuu Lee ${ }^{2,3}$, Runsheng Wang ${ }^{1}$, Yangyuan Wang ${ }^{1,3}$ \\ ${ }^{1}$ Key Laboratory of Microelectronic Devices and Circuits (MOE), Institute of Microelectronics, Peking University, Beijing 100871, China \\ ${ }^{2}$ Semiconductor Manufacturing International Corporation (SMIC), Shanghai 201203 and Beijing 100176, China \\ ${ }^{3}$ Innovation Center for MicroNanoelectronics and Integrated System, Beijing 100871, China \\ *Email: ruhuang@pku.edu.cn
}

\begin{abstract}
In this paper, using DTMOS as an effective solution of RTN suppression without device/circuit performance penalty is proposed and demonstrated for the first time, with experimental verification and circuit analysis. The experiments show that RTN amplitude is greatly reduced in DTMOS mode, which is even better than the body-biasing technique of FBB, due to the efficient dynamic modulation mechanism. Circuit stability and performance degradation induced by RTN are much improved in the design using DTMOS. New characteristics of RTN physics in DTMOS are also observed and studied in detail. The results are helpful to the robust and reliable device/circuit co-design in future nano-CMOS technology.
\end{abstract}

\section{Introduction}

Recently, the random telegraph noise (RTN) has been drawing much attention as a significant source of dynamic variability [1-6]. Because it can lead to large $\mathrm{V}_{\mathrm{TH}}$ (or $\mathrm{I}_{\mathrm{D}}$ ) degradation and cause transient probabilistic failure of digital circuits due to its stochastic nature as we have demonstrated [5-7], RTN will narrow the tight design margin in nano-CMOS technology. Several attempts through process adjustment are reported (e.g., buried channel), but with device performance degradation and/or ineffective for the newborn-trap induced RTN during circuit aging. Therefore, effective RTN suppression technique without performance penalty emerges as an urgent demand for device/circuit co-design.

In this paper, we propose, for the first time, to use the dynamic threshold MOS (DTMOS) operation mode as an effective method to suppress RTN without intrinsic device/circuit performance loss. The experimental results show that RTN amplitude is greatly reduced in DTMOS mode, and new characteristics of RTN time constants are found in DTMOS. The effectiveness of RTN suppression in DTMOS is also demonstrated in circuit analysis in terms of performance degradation and transient variation.

\section{Strategy for RTN Suppression at} Device/Circuit Level

Generally for variability reduction, apart from process adjustment and system redundancy, body-biasing technique (FBB/RBB) has been widely used at the device/circuit level [8]. Recently, it is demonstrated that FBB is helpful for reducing flicker noise [9]. In this work we further examine whether FBB is also suitable to RTN suppression for the first time. Meanwhile, considering that DTMOS is more efficient in dynamic modulation, we propose to use it as a more effective way for device/circuit co-design against RTN. Since no study on the RTN characteristics of DTMOS has been reported, the new features of RTN physics will be investigated by experiments in this work as well.

\section{Experimental Demonstration}

A. Devices and RTN characterization. The devices used in this work are with $28 \mathrm{~nm}$ high-k/metal-gate (HKMG) technology. Three types of device operation mode and the corresponding measured $\mathrm{I}_{\mathrm{D}}-\mathrm{V}_{\mathrm{G}}$ curves are shown in Fig. 1, including MOS, FBB MOS and DTMOS modes. It is worth noting that, both the RTN amplitude $\left(\Delta \mathrm{V}_{\mathrm{TH}}\right.$ or $\left.\Delta \mathrm{I}_{\mathrm{D}} / \mathrm{I}_{\mathrm{D}}\right)$ and time constants are crucial for accurate evaluating the impacts of RTN in circuits. The circuit static performance degradation depends on the RTN amplitude, while the time constants determine the dynamic variability and transient failure probability in digital circuits. Fig. 2 shows the typical experimental results of RTN in MOS, FBB and DTMOS modes, which have evident reduction in the aspect of amplitude. The deviation of time constants can also be found in Fig. 2(e) (h).

B. RTN amplitudes reduction in DTMOS mode. Fig. 3 shows the extracted RTN amplitudes at different gate voltages in MOS mode, FBB mode and DTMOS mode. The RTN reduction in both FBB and DTMOS modes can be observed, but DTMOS mode has the largest amount of reduction. Fig. 4 shows that a maximum RTN amplitude reduction of $58 \%$ can be obtained in DTMOS mode in this work. The similar phenomenon have also been observed with the SiON gate dielectric devices (not shown here).

To get a clear understanding of the RTN reduction mechanisms in FBB and DTMOS modes, it is useful to plot $\Delta I_{D} / I_{D}$ with gate overdrive $\left(V_{G^{-}}-V_{T H}\right)$, for normalizing the effect of body biasing. As shown in Fig. 5(a), the $\Delta \mathrm{I}_{\mathrm{D}} / \mathrm{I}_{\mathrm{D}}$ curves of FBB mode converge with the MOS curve, which indicates that the RTN reduction in FBB mode results from 
the body-bias induced $\mathrm{V}_{\mathrm{TH}}$ shift only. However, the curve of DTMOS mode is still far below the others as expected, because of the different operation mechanism. For a given oxide trap, its RTN amplitude is mainly determined by the inversion charge (see equation 4), which is proportional to $\left(\mathrm{V}_{\mathrm{GS}}-\mathrm{V}_{\mathrm{TH}}\right)$ and the gate capacitance. The equivalent gate capacitance in DTMOS is larger and still increases with increasing $\mathrm{V}_{\mathrm{G}}$ after strong inversion [10], rather than keeping constant as in MOS and FBB modes. Large inversion charge in DTMOS leads to reduced RTN amplitude. Alternatively, it can also be interpreted as follows. Two boundary values of the $\mathrm{V}_{\mathrm{TH}}$ shift are marked as $\Delta \mathrm{V}_{\mathrm{TH} 1}$ and $\Delta \mathrm{V}_{\mathrm{TH} 2}\left(\Delta \mathrm{V}_{\mathrm{TH} 1}<\Delta \mathrm{V}_{\mathrm{TH} 2}\right)$ in Fig. 5(b). It can be found that the left side of the $\mathrm{V}_{\mathrm{TH} 1}$-shifted curve and the right side of the $\mathrm{V}_{\mathrm{TH} 2}$-shifted curve are merged into the MOS curve [Fig. 5(c)], which indicates that the RTN reduction results from the dynamic $\mathrm{V}_{\mathrm{TH}}$ modulation effect of DTMOS.

\section{New observations on time constants in DTMOS mode.}

Fig. 6 shows the extracted capture time constant $\left(\tau_{\mathrm{C}}\right)$ and emission time constant $\left(\tau_{\mathrm{E}}\right)$ of RTN, and reveal the same $\mathrm{V}_{\mathrm{G}}$ dependence of $\tau_{\mathrm{C}}$ in both MOS and DTMOS mode. However, a very different $\mathrm{V}_{\mathrm{G}}$ dependence of $\tau_{\mathrm{E}}$ is observed in DTMOS mode. As shown in Fig. 8\&9, within the RTN test window, the opposite trend of the $\mathrm{V}_{\mathrm{G}}$ dependence of surface potential (and thus $E_{C \text {-eff }}$ ) and $\mathrm{E}_{\mathrm{T}}$ contribute to the non-monotonic trend of $\tau_{\mathrm{E}}$ (see Eq. 1). Besides $\tau_{\mathrm{C}}$ and $\tau_{\mathrm{E}}$ themselves, it is even more important to focus on $\tau_{\mathrm{C}} / \tau_{\mathrm{E}}$, which reflects the trap spatial position $\left(x_{\mathrm{T}} / \mathrm{T}_{\mathrm{OX}}\right)$ by equation (2) and also indirectly determines the trap occupancy rate [5-7]. For DTMOS mode, $\tau_{\mathrm{C}} / \tau_{\mathrm{E}}$ in Fig. $7(\mathrm{a})(\mathrm{b})(\mathrm{d})$ have non-monotonic $\mathrm{V}_{\mathrm{G}}$ dependence, and the corner points exhibit a linear relationship with $\mathrm{x}_{\mathrm{T}} / \mathrm{T}_{\mathrm{OX}}$ (Fig. 10). An effective surface Fermi level $E_{F-e f f}$ is introduced here in DTMOS mode as shown in Fig. 8 due to the splitting of Fermi energy levels at the bulk and surface, and it decreases as $V_{G}$ increasing in nMOS devices, with more rapid speed for larger $V_{G}$. Therefore, with the modified model in equation (3), the new observations of the $\mathrm{V}_{\mathrm{G}}$ dependence of $\tau_{\mathrm{C}} / \tau_{\mathrm{E}}$ in DTMOS can be well explained, and can also be predicted for the range out of the RTN test windows as shown in Fig. 11.

Fig. $12 \& 13$ shows the statistical $V_{G}$ dependence of $\tau_{\mathrm{C}} / \tau_{\mathrm{E}}$ and the relationship between the Lorentzian power spectral density (PSD) of single RTN and $\tau_{\mathrm{C}} / \tau_{\mathrm{E}}$. According to the results above, the distribution of Lorentzian PSD of multiple traps can be obtained as shown in Fig. 14, and it can be found that the Lorentzian PSD of most of the traps in DTMOS mode are smaller than MOS mode, which suggests DTMOS mode as an effective solution against RTN.

\section{Effectiveness of RTN Suppression in Circuits Design}

Based on the experimental results, transient circuit simulation of RTN is performed on our recently developed platform [7]. For the DTMOS mode, $\tau_{\mathrm{C}} / \tau_{\mathrm{E}}$ are categorized into two types based on the comparison results between MOS and DTMOS mode (Fig. 15). Fig. 16 shows the ring oscillator (RO) adopted for simulation as the representative digital circuit and the corresponding eye diagram with the RTN effects. The $\mathrm{V}_{\mathrm{G}}$ dependence of amplitude and time constants are included with the models in Table I.

Fig. $17 \& 18$ show the jitter and the mean shift of center frequency in a single RO operating at three modes, indicating the DTMOS mode as the best solution against RTN in the aspects of both variation and degradation. The jitters of RO under different RTN conditions are compared in Fig. 19, which suggests it important to consider the new properties of time constants in DTMOS mode. It shows that the suppression of type 2 RTN in DTMOS is not as good as type 1 (but still better than FBB). But fortunately, type 2 RTN is actually less than $20 \%$ in reality (see Fig. 12). As for the realistic applications, it is important to pay attention to the VDD dependence of the static degradation and the dynamic variability. Fig. 20 shows the jitters under different VDD in three modes, and the $\Delta f / f$ and $\sigma(\mathrm{RO}$ jitter) are extracted as show in Fig. 21, which suggest that for all these modes, the degradation and variability induced by single RTN increase with reduced VDD, and DTMOS is still the best mode. Therefore, as for low-power robust design against RTN, DTMOS is the best choice.

\section{Summary}

An effective method to suppress RTN without compromising intrinsic performance is proposed in this paper for the first time, which is demonstrated with the experiments and circuit analysis. It is found that using DTMOS mode can greatly suppress RTN impacts on device and circuits, and is even better than FBB technique. The results are useful for the robust and reliable design against RTN in the future.

\section{Acknowledgement}

This work was partly supported by the 973 Projects (2011CBA00601), NSFC (61106085), and National S\&T Major Project (2009ZX02035-001).

\section{References}

[1] T. Matsumoto, et al., IEDM, 2012, pp.581-584. [2] H. Miki, et al., IEDM, 2012, pp.450-453. [3] N. Tega, et al., VLSI, 2009, pp.50-51. [4] S. Realov, et al., IEDM, 2010, pp.624-627. [5] J. Zou, et al., VLSI, 2012, pp.139-140. [6] J. Zou, et al., VLSI, 2013, pp.186-187. [7] R. Wang, et al., IEDM, 2013, pp.834-837. [8] S. Narendra, et al., IEEE JSSC, vol. 38, no. 5, pp. 696-701, 2003. [9] Arnoud P. van der Wel, et al., IEEE JSSC, vol. 42, no. 3, pp.540-550, 2007. [10] R. Huang, et al, Solid-State Electronics, vol. 47, no. 8, 2003. 


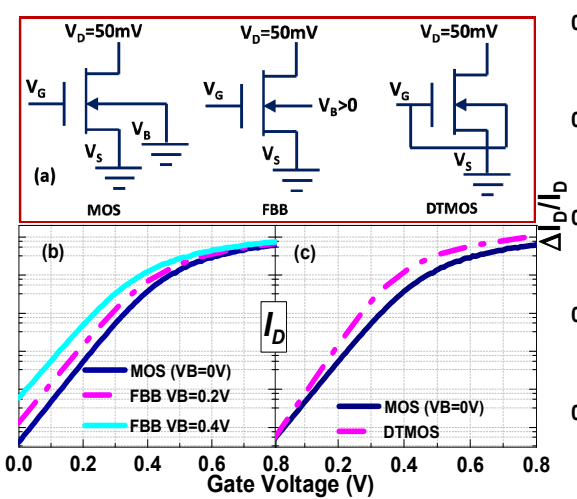

Fig. 1 (a) Bias conditions for measurements in MOS, FBB and DTMOS modes. Comparisons of $\mathrm{I}_{\mathrm{D}}-\mathrm{V}_{\mathrm{G}}$ curves in these three modes are shown in (b) \& (c) respectively.
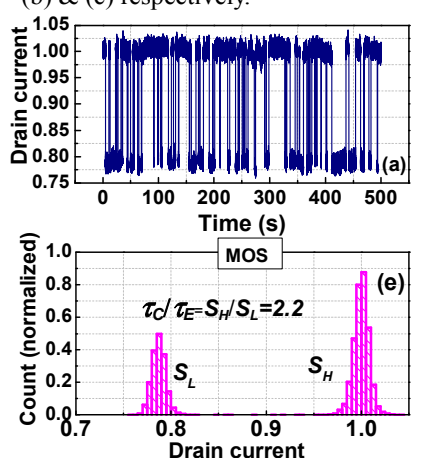
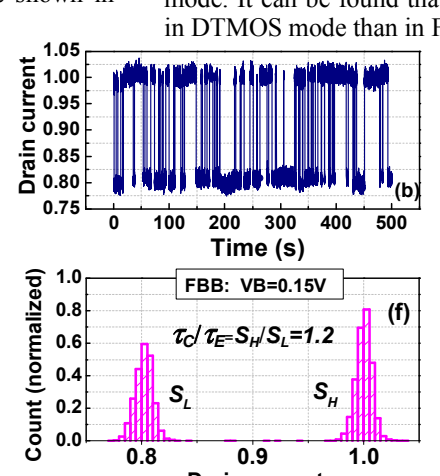

(a)

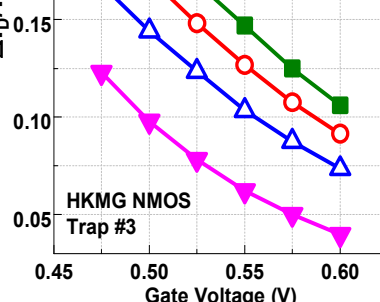

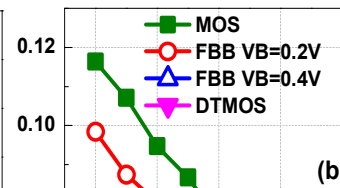

(b)

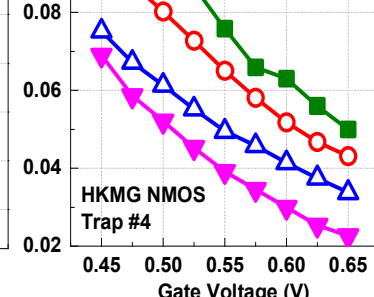

Gate Voltage (V)

Fig. 3 The experimental results of RTN amplitudes in two HKMG NMOS devices in MOS mode, FBB mode and DTMOS mode. It can be found that amplitudes can be better suppressed in DTMOS mode than in FBB mode.

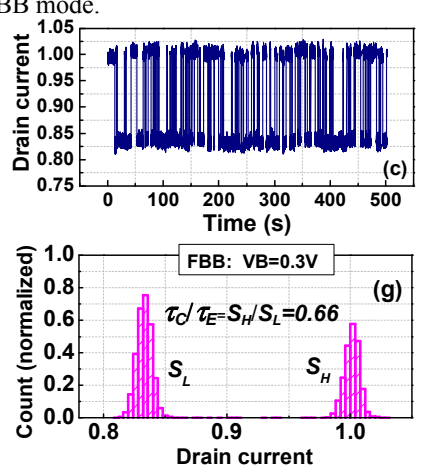

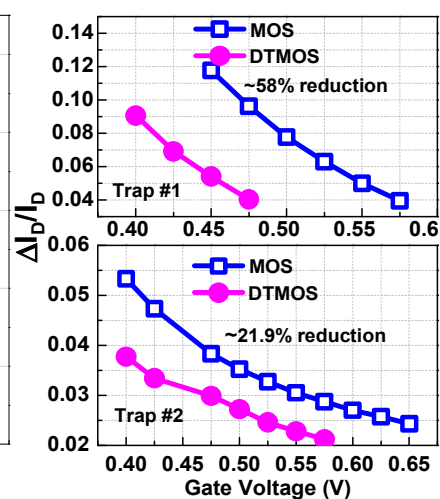

Fig. 4 Comparison of the RTN amplitudes in MOS mode and DTMOS mode. The amplitude reduction effect of DTMOS mode is further confirmed.

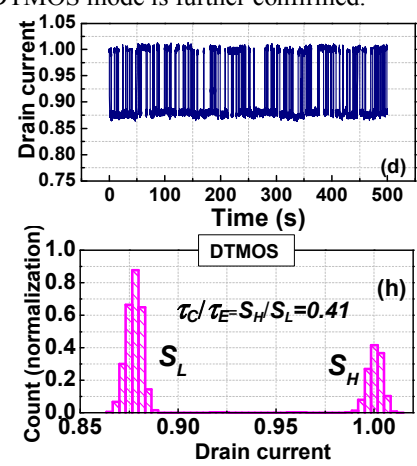

Fig. 2 (a) (b) (c) (d) Raw data of the measured RTN induced by the same trap in MOS, FBB and DTMOS modes. Note: the drain currents are normalized to the high current states. (e) (f) (g) (h) Histogram of the measured drain currents. The ratio of the percentages in high and low states corresponds to the $\tau_{\mathrm{C}} / \tau_{\mathrm{E}}$ as shown.
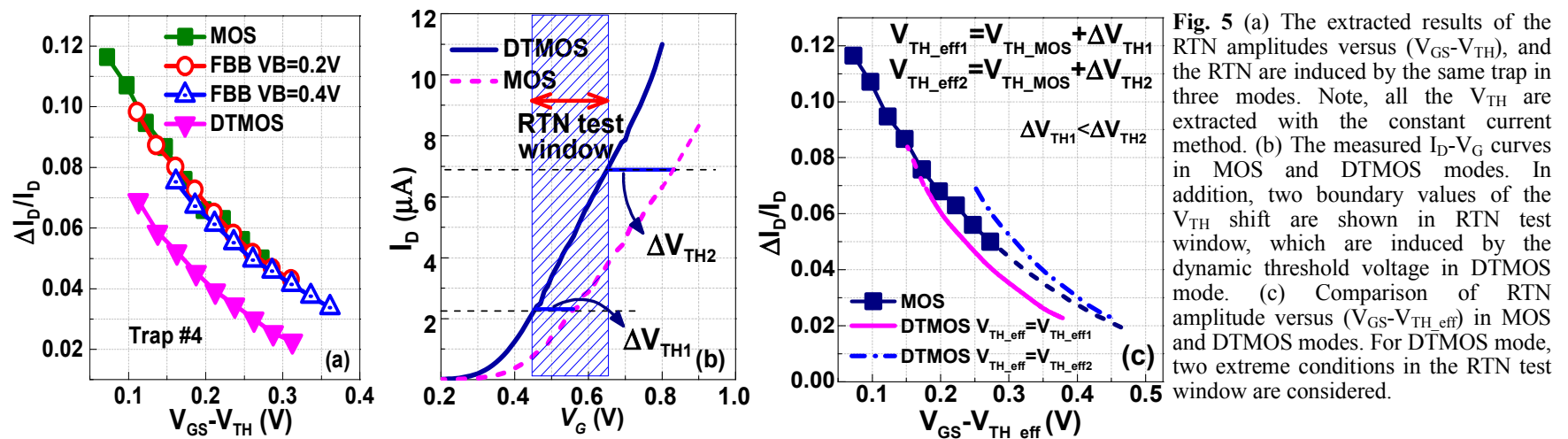

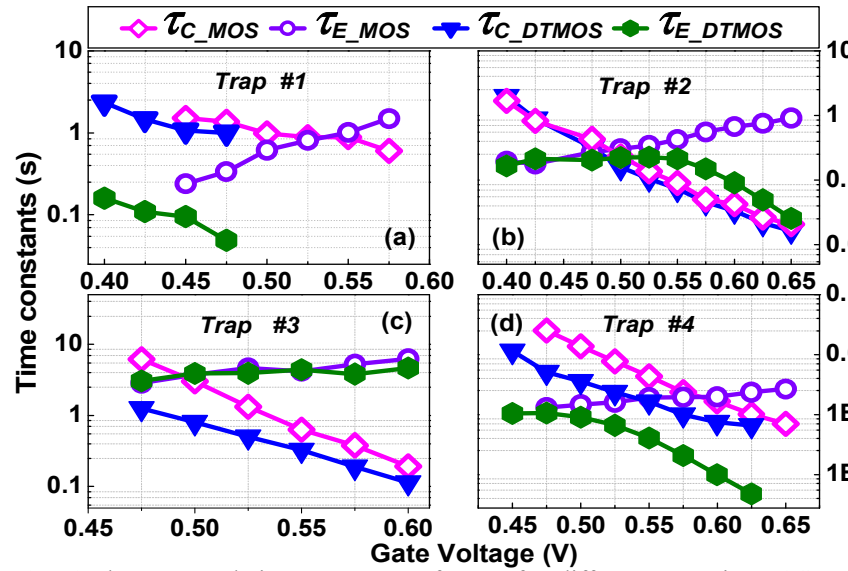

Fig. 6 The extracted time constants of RTN for different traps in MOS and DTMOS modes. An observed $\mathrm{V}_{\mathrm{G}}$ dependence of $\tau_{\mathrm{E}}$ in DTMOS mode is different from that in MOS mode.

Table I Some key equations used in analyzing the time constants and amplitude of RTN

$$
\begin{aligned}
& \text { (1) RTN capture \& emission time constants : } \\
& \tau_{c}=\frac{1}{\sigma v_{t h} n} \quad \tau_{e}=\frac{1}{\sigma v_{t h} N_{c} \exp \left[\left(E_{T}-E_{C_{-} e f f}\right) / k T\right]} \\
& \frac{\tau_{c}}{\tau_{e}}=\exp \left(\frac{E_{T}-E_{F_{-} e f f}}{k T}\right) \quad E_{T}=E_{T 0}-q \frac{x_{T}}{T_{O X}}\left(V_{g s}-V_{F B}-\varphi_{s}\right) \\
& \ln \frac{\tau_{c}}{\tau_{e}}=-\frac{1}{k T}\left[\begin{array}{c}
\left(E_{C o x}-E_{T 0}\right)-\left(E_{C_{-} e f f}-E_{F_{-} e f f}\right)-\Phi_{0} \\
+q \varphi_{s}+q \frac{x_{T}}{T_{O X}}\left(V_{g s}-V_{F B}-\varphi_{s}\right)
\end{array}\right]
\end{aligned}
$$

(2) For MOS:

(3) For DTMOS :

$$
\begin{gathered}
\frac{d\left(\ln \tau_{c} / \tau_{e}\right)}{d V_{g s}}=-\frac{q}{k T} \frac{x_{T}}{T_{O X}} \quad \text { when } V_{g s}-V_{T H}>0 \\
\frac{d\left(\ln \tau_{c} / \tau_{e}\right)}{d V_{g s}}=-\frac{1}{k T}\left(\frac{q x_{T}}{T_{O X}}-\frac{d\left(E_{C_{e f f}}-E_{F_{e f f}}\right)}{d V_{g s}}+q\left(1-\frac{x_{T}}{T_{O X}}\right) \frac{d \varphi_{s}}{d V_{g}}\right. \\
\text { dence: } \quad \frac{\Delta I_{D}}{I_{D}}=\frac{4 r^{2}}{W L-2 r(L-2 r)} r=\sqrt{\frac{q}{Q} \frac{d_{1}}{d_{1}+d_{2}}} \\
Q=C_{O X}\left(V_{G S}-V_{F B}-\varphi_{S}\right)-q N_{b} x_{d e p}
\end{gathered}
$$$$
\text { (4) RTN amplitude dependence: } \frac{\Delta I_{D}}{I_{D}}=\frac{4 r^{2}}{W L-2 r(L-2 r)} r=\sqrt{\frac{q}{Q} \frac{d_{1}}{d_{1}+d_{2}}}
$$ 


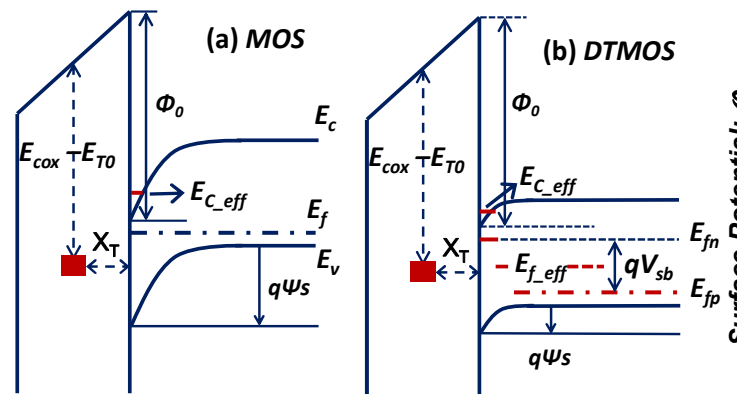

Fig. 8 (a) The schematic energy band diagram of NMOS. (b) The schematic energy band diagram of a n-type DTMOS. It is worth noting that $E_{C_{-} e f f}$ is introduced due to the quantum confinement effect, and the effective Fermi energy level $E_{f-e f f}$ decreases with $V_{\mathrm{G}}$
increasing as a result of the increasing split of quasi-Fermi energy levels between the bulk and surface.

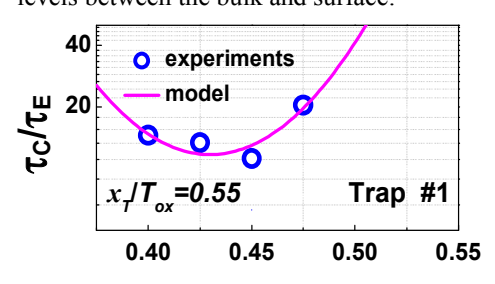

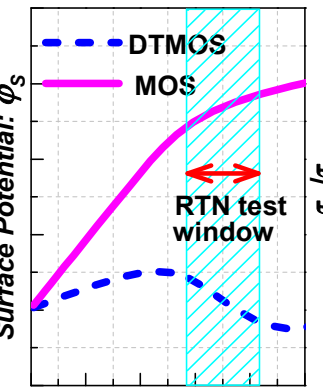

Gate Voltage (V)
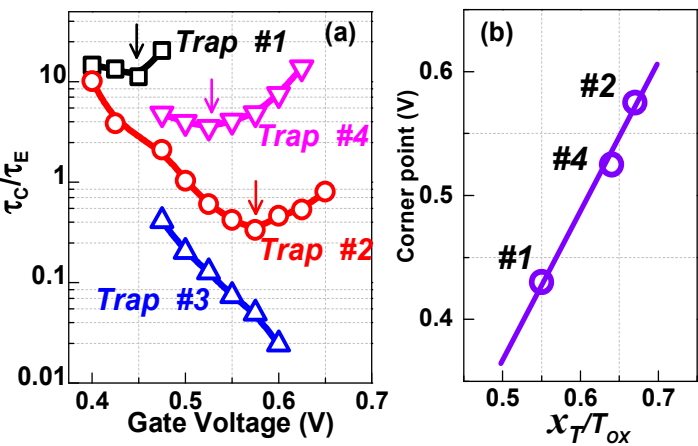

Fig. 9 The simulated $V_{G}$ Fig. 10 (a) Comparison of the $V_{G}$ dependence of $\tau_{C} / \tau_{E}$ in each trap. dependence of the Note that, the corner points are different, and it can be observed surface potential in MOS that the positions of the corner point have a linear relationship with mode and DTMOS the corresponding $x_{T} / T_{O X}$, as shown in (b).
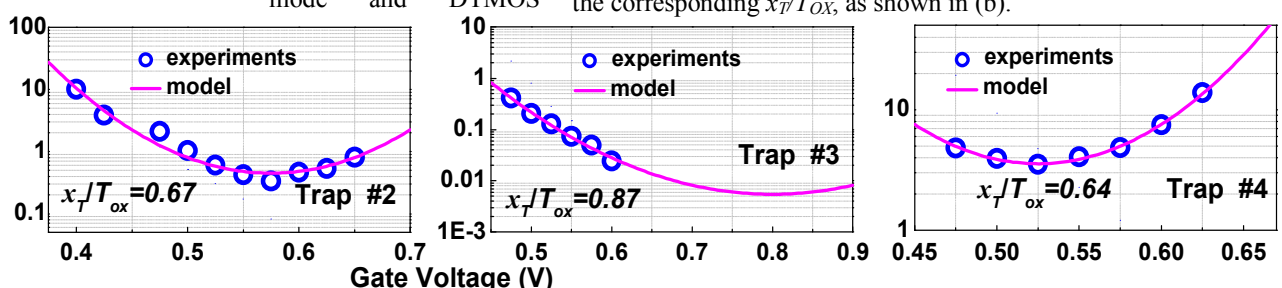

Fig. 11 The predicted values (line) of $\tau_{\mathrm{C}} / \tau_{\mathrm{E}}$ of different traps in DTMOS mode according to the improved model. For trap \#3, the corner point can be predicted at about $0.8 \mathrm{~V}$. With this method, more comparisons can be done for traps located at different positions in gate dielectric between MOS mode and DTMOS mode.

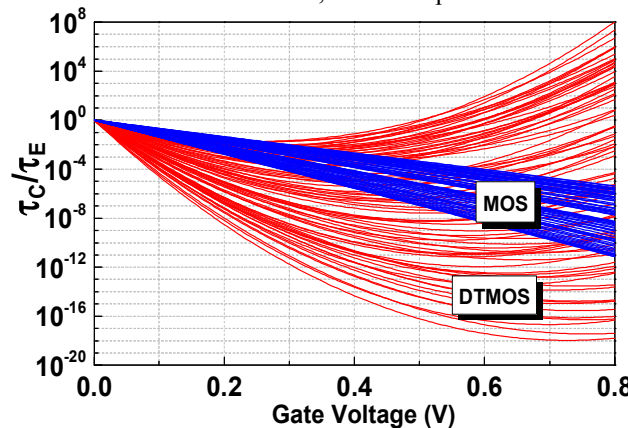

Fig. 12 Statistical $V_{G}$ dependence of $\tau_{C} / \tau_{E}$ predicted according to equation (2) \& (3) in MOS mode and (b) Illustration of the relationship between DTMOS modes. Note that, $\tau_{\mathrm{C}} / \tau_{\mathrm{E}}$ has been normalized to Lorentzian PSD and $\tau_{\mathrm{C}} / \tau_{\mathrm{E}}$
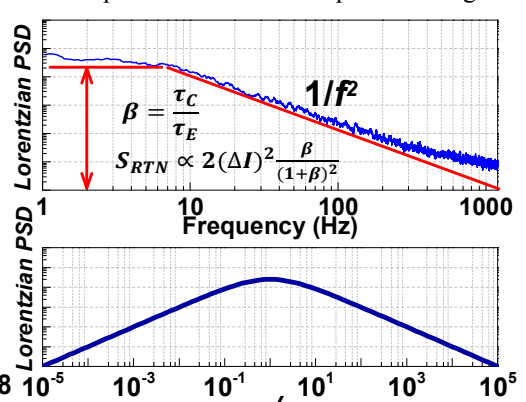

$\tau_{c} / \tau_{E}^{10}$

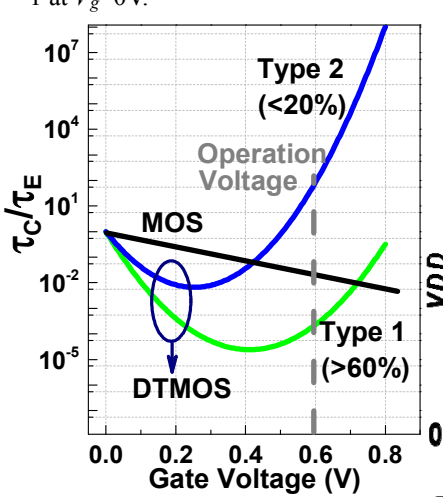

Fig. 15 For DTMOS mode, RTN are categorized into two types according to the trend of $\tau_{\mathrm{c}} / \tau_{\mathrm{s}}$ changing with gate voltage.

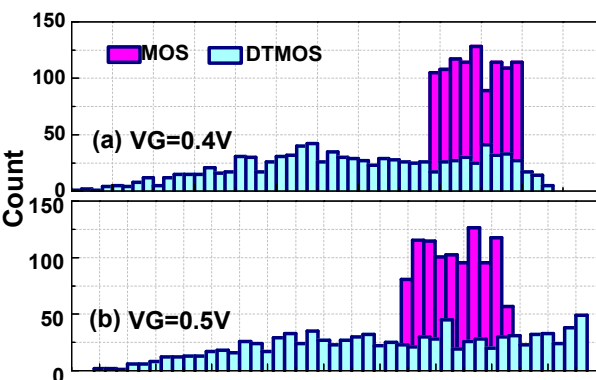

Lorentzian PSD (log) (a.u.)

Fig. 14 Comparison of the distributions of

Lorentzian PSD induced by different traps in MOS

mode and DTMOS mode.

DTMOS
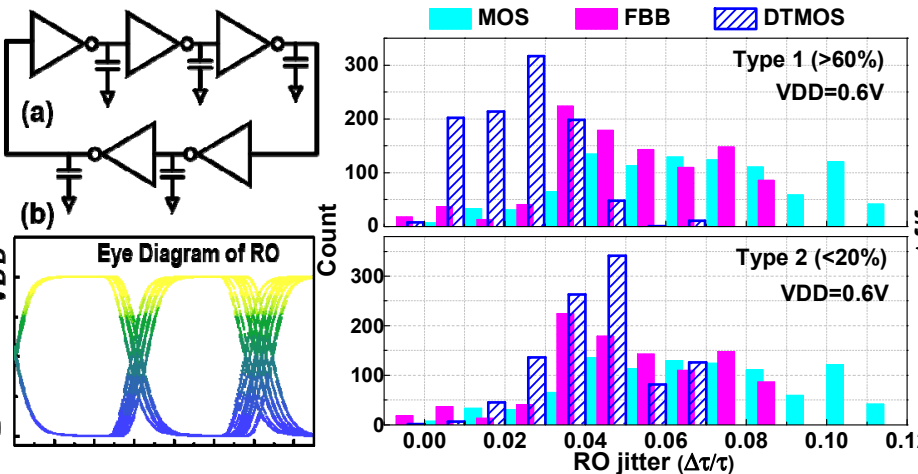

Fig. 17 The simulation results of the RO jitter distributions with the impacts of (a) type 1 and (b) type 2 traps in DTMOS mode. All of them are compared with the jitter distributions in the MOS

mode and FBB mode.

DTMOS-2: $w /$ impacts of type 2 RTN

Fig. 16 (a) The 5-stage RO adopted for simulation in this work. (b) Typical eye diagram of RO with the effects of RTN

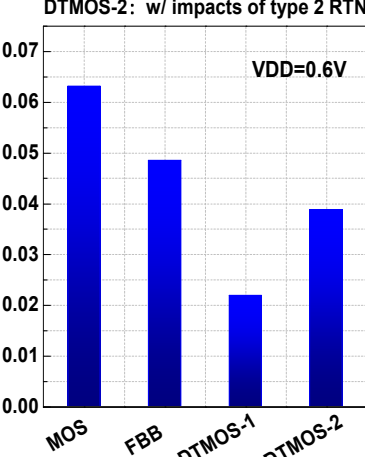

Fig. 18 Comparison of the centric frequency degradation impacted by RTN in different modes, including MOS mode, FBB mode and DTMOS mode.

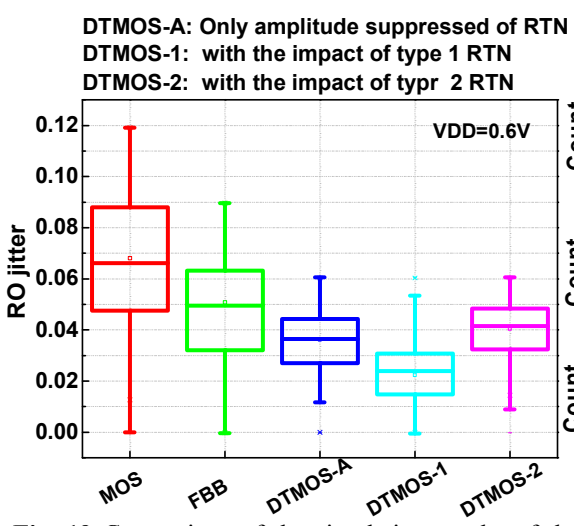

Fig. 19 Comparison of the simulation results of the jitter distributions in different modes.

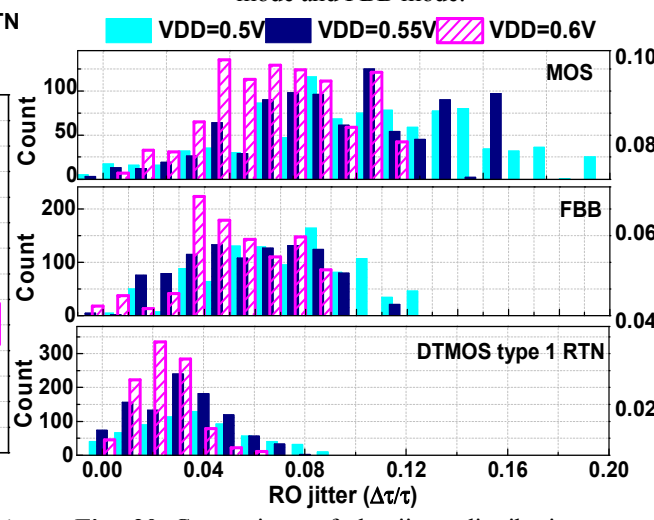

Fig. 20 Comparison of the jitter distributions under different VDD in these three modes. $\rightarrow$ MOS $\triangle$ FBB $\triangle$-DTMOS-1 $\checkmark-$ DTMOS-2

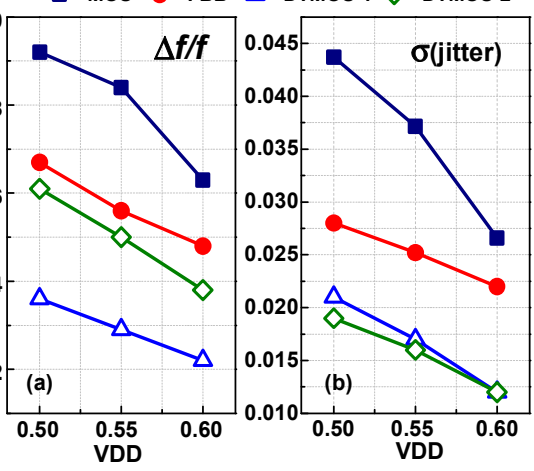

Fig. 21 The simulation results of the VDD dependence of $\Delta f / f$ and $\sigma(j i t t e r)$ in different modes. 